\title{
SINGLE DOSE PRE-OPERATIVE ANTIBIOTIC IN LICHTENSTEIN MESH HERNIOPLASTY
}

\author{
Ankur Sharma1, Rizwan Ullah Khan², Goonj Johri ${ }^{3}$ \\ ${ }^{1}$ Assistant Professor, Department of General Surgery, IIMS\&R, Integral University, Lucknow. \\ ${ }^{2}$ Assistant Professor, Department of General Surgery, IIMS\&R, Integral University, Lucknow. \\ ${ }^{3}$ Assistant Professor, Department of General Surgery, ELMC, Lucknow.
}

\begin{abstract}
BACKGROUND

Inguinal hernia repair is one of the commonest general surgical procedures worldwide. Although considered to be a clean surgical procedure, post-operative Surgical Site Infection (SSI) can complicate a mesh repair and add to significant patient morbidity and anxiety to the surgeon. Prolonged use of post-operative antibiotics is not recommended anymore, but many surgeons still continue to use prolonged post-operative antibiotics which add to the cost of treatment and also increase chances of side effects and toxicity to the patient.
\end{abstract}

\section{METHODS}

The study was done as a retrospective data analysis for patients who had received single dose of pre-operative antibiotic.

\section{RESULT}

Single dose pre-operative antibiotic is effective in reducing SSI after an elective hernia surgery and prolonged doses of antibiotics may not be necessary.

\section{KEYWORDS}

Single Dose, Antibiotic, Mesh Hernioplasty, Surgical Site Infection.

HOW TO CITE THIS ARTICLE: Sharma A, Khan RU, Johri G. Single dose pre-operative antibiotic in Lichtenstein mesh hernioplasty. J. Evolution Med. Dent. Sci. 2016;5(55):3737-3740, DOI: 10.14260/jemds/2016/857

\section{INTRODUCTION}

Hernia is defined as abnormal protrusion of viscera or part of viscera through an abnormal opening in the wall of its containing cavity. Of all the hernias, inguinal hernias are the most common variety. More than 100 types of surgeries have been described for repair of inguinal hernia. Of these, Lichtenstein mesh hernioplasty and laparoscopic Hernia surgery are currently popular. The Lichtenstein technique is a tension free repair of weakened inguinal floor with use of polypropylene mesh anchored to the inguinal region.(1) Hernia repair surgery is one of the most commonly performed general surgery procedure in current era.(2) Any modification to such high volume surgery or strategies for reduction in morbidity of the procedure or reduction in cost of hernia treatment will have far reaching impact all over the world.

Post-operative Surgical Site Infection (SSI) after inguinal hernia surgery is reported to be between 0-9\%.(3) Many of these infections are often recognized in the outpatient setup, after discharge from the hospital as more and more number of cases are done as day care procedures. ${ }^{(4)}$

The risk of Surgical Site Infection (SSI) increases with introduction of a prosthesis in the body, due to alteration in body defence mechanisms.

The low rate of SSI is the main argument against routine antibiotic prophylaxis for hernia surgery.(5) However, since a

Financial or Other, Competing Interest: None.

Submission 29-05-2016, Peer Review 29-06-2016,

Acceptance 04-07-2016, Published 09-07-2016.

Corresponding Author:

Dr. Ankur Sharma,

Assistant Professor,

Department of General Surgery, IIMS\&R,

Integral University, Dasauli,

Kursi Road,

Lucknow-226026.

E-mail: ankurvashishth@gmail.com

DOI: $10.14260 /$ jemds/2016/857 prosthetic mesh is used and mesh infection can be potentially disastrous, many surgeons prefer to use antibiotic prophylaxis in peri-operative period.

SSIs may prolong the hospital stay by one week and increase the treatment costs by more than $20 \% .^{(6)}$

Antibiotics play an important role in prevention of SSIs. Such efficacy of antibiotics in preventing SSIs is significant, but it should not be a substitute for compromise in surgical asepsis, technique of surgery or haemostasis. As early as 1961, Burke recommended use of prophylactic antibiotics in preoperative period to reduce the wound infections. (7) Such prophylactic use of antibiotics was again recommended by various authors and laid ground for routine use of prophylactic antibiotics to reduce wound infection rates. $(8,9)$

The duration of this peri-operative antibiotic coverage varies widely from centre to centre and surgeon to surgeon. The first randomized control trial on the role of antibiotic prophylaxis in mesh hernioplasty in 2001, advocated the use of prophylactic antibiotics.(10) However, a Cochrane metaanalysis done in 2003 opined that antibiotic prophylaxis in elective inguinal hernia repair cannot be recommended or discarded and that further studies were needed, particularly in cases with mesh repair.(11)

Cephalosporins are generally the preferred antibiotics for peri-operative prophylaxis agents for many surgical procedures. They have the capability to target the probable pathogens that may cause the infection in post-operative period. Use of broad-spectrum antibiotics is discouraged, because they may help in development of antimicrobial resistance.(12)

Amoxicillin-clavulanic acid combination is another antibiotic, which is used for antibiotic prophylaxis including hernia repair.(13,14) We used single dose of amoxicillinclavulanic acid for antibiotic prophylaxis in our study as per existing department policy. 


\section{MATERIALS AND METHODS}

The aim of this study was to document and report incidence of wound infection in clean hernia surgeries at our centre. The study also aimed to document, if routine use of single dose antibiotic is feasible in our setting.

The study was done as retrospective data analysis of patients who underwent Lichtenstein mesh hernioplasty at our Hospital, IIMS\&R, Lucknow. Our hospital predominantly caters to suburban and rural population. As per department protocol, Mesh hernioplasty is offered to all patients with inguinal hernia with age more than 25 years and between the ages of 15 to 25 years for patients who have poor abdominal tone.

Study period was from March 2013 till Feb 2015, for which the data was collected and analysed.

\section{Inclusion Criteria}

All patients who underwent Lichtenstein mesh hernioplasty and had received only one dose of prophylactic antibiotic were included in the study.

\section{Exclusion Criteria}

1. Emergency hernia surgery for obstruction or strangulation.

2. Recurrent hernias.

3. Immunocompromised patients like diabetes, HIV or steroid treatment.

4. Patients who underwent herniorrhaphy.

5. Patients who had received pre-operative antibiotics in last 7 days due to any indication were also excluded.

\section{Surgical Technique}

Lichtenstein tension free mesh hernioplasty was carried out in all the patients. Cases were done under either in general, spinal or local anaesthesia depending on surgeon preference.

The skin was shaved one day previous to surgery and preoperative skin was prepared with $10 \%$ povidone-iodine solution. Standard surgical technique for mesh repair was followed. Prolene mesh was used and anchored with 2-0 prolene suture. Skin was closed with 3-0 polyamide black. No drains were used.

Single dose of antibiotic, $1.2 \mathrm{gm}$ of amoxicillin and clavulanic acid combination was administered 30 mins. before the proposed skin incision. No post-operative antibiotic was given.

At the end of surgery wound was dressed with standard sterile dressing, which was removed 48 hours after the surgery and the wound was inspected. The patients were discharged either after the first wound inspection or after suture removal as per patient's choice.

\section{End Points:}

There were two end points of the study.

The first end point of the study was superficial wound infection either in the form of wound erythema, cellulitis, purulent discharge or wound dehiscence any time after surgery till suture removal.

The first wound inspection was done at 48 hours after surgery and then again at the time of suture removal.

The second end point was deep wound infection manifested in the form of mesh infection within one year from the date of surgery.

\section{The following parameters were studied from the Patients'} files'

1. Demographic details including age, sex, comorbid conditions, type of hernia and duration of symptoms.

2. Surgical details including type of hernia, technique of procedure done, type of anaesthesia and duration of surgery.

3. Antibiotic administered.

4. Incidence of wound infection and would culture report if applicable.

\section{RESULTS}

During the study period, mesh hernioplasty was done in 196 patients. Of these, only 154 patients fulfilled the inclusion criteria and had complete one-year follow-up and were included in the study.

Wound infection was noted in 5 patients $(3.2 \%)$.

There was no case of mesh infection during the study period.

\section{Patient Data}

The age range was 23 to 76 years and mean age was 40.2 years.

\begin{tabular}{|c|c|}
\hline Age Group & Number \\
\hline $21-30$ Years & 28 \\
\hline $31-40$ Years & 49 \\
\hline $41-50$ Years & 42 \\
\hline $51-60$ Years & 26 \\
\hline 61-70 Years & 6 \\
\hline 71-80 Years & 3 \\
\hline \multicolumn{2}{|c|}{ Table 1: Table showing Age } \\
Distribution of Patients \\
\hline
\end{tabular}

- $\quad$ There were 150 males and 4 females.

- American Society of Anaesthesiologists (ASA) Category:

- There were 102 patients of ASA 1 category, 36 patients of ASA 2 and 16 patients of ASA 3 category.

- There were 70 patients with right inguinal hernia, 62 patients with left hernia and 22 patients with bilateral hernia.

- Duration of surgery ranged from 38 mins. to 78 mins. and mean duration of surgery was 44.2 mins.

- Wound culture isolated Staph. aureus in 2 patients, whereas other patients were culture negative.

\section{DISCUSSION}

Surgical Site Infections (SSI) account for 14 to $18 \%$ of all health care related infections and are presumed to be third most common nosocomial infection.(15,16) Data pertaining to SSIs is complicated by the mixed and wide nature of their presentation and also by inter-person variations in reporting. SSIs also vary from centre to centre, surgeon to surgeon, the type of surgery performed and the hospital setting. The highest risk of SSIs is noted in large teaching hospital with beds more 500 , followed by smaller teaching hospitals and the least incidence in non-teaching hospitals.(17)

SSIs are not only a source of morbidity to the patient and anxiety to the surgeon, but they are also responsible for increased financial burden. Such increased financial burden can be particularly disturbing to patients with limited financial resources. SSIs also increase the hospital stay for patients. Also, SSIs increase the risk of death in hospitalised patients. 
Peri-operative antibiotics have been shown to decrease the incidence of SSIs. $(18,19)$

Factors which increase the risk of SSI include patient related factors and procedure related factors. Patient related factors include age, nutrition, immunocompromised states like diabetes mellitus, obesity and length of preoperative stay whereas the procedure related factors include type of surgery, duration of procedure, antibiotic prophylaxis, skin antisepsis, operative room ventilation, sterilization of surgical instruments, use of prosthesis during surgery, surgical drains and surgical technique. $(20,21)$

Although western literature frequently reports incidence of SSIs between $0.5-15 \%$, studies reported from India show a higher incidence of SSIs between 23-38\%. This wide variation between these maybe in part due to different factors like including patient's nutritional status, sterile precautions during the procedure and the setting of hospital.(22)

The risk of SSI also depends on whether the procedure is clean, clean-contaminated, contaminated or dirty, based on their standard definitions of these terms as defined in 1964.(23)

Perioperative antibiotics are routinely recommended for variety of surgical procedures to prevent surgical site infections. Optimal antimicrobial agents for prophylaxis should be bactericidal, nontoxic, inexpensive and active against the typical pathogens that can cause surgical site infection postoperatively. (20)

Antibiotic prophylaxis is used to prevent SSI, but their use should be limited to avoid excess cost, toxicity and development of antibiotic resistance. Prolonged use of antibiotics is associated with increased healthcare cost, increased incidence of undesirable side effects and also increases the chances of antibiotic resistance. The duration of AP for most procedures should not exceed 24 hours with the exception of cardiac surgeries, in which antibiotics may be continued for up to 48 hours. $(24,25,26,27)$

\section{Wound Infection}

It is defined as following.(28)

- Purulent discharge from the incision site with or without positive culture and sensitivity report.

- Non-purulent discharge from incision site with positive culture and sensitivity report.

- Incision site deliberately opened by surgeon in the presence of at least one of the following signs or symptoms of infection: pain or tenderness, localized swelling, redness or heat.

- Diagnosis of infection by the surgeon.

SUMMARY OF THE DEFINITIONS OF SUPERFICIAL AND DEEP SSIS SUPERFICIAL INCISIONAL SURGICAL SITE INFECTIONS. ${ }^{28}$

Superficial Incisional Surgical Site Infections must meet the following two Criteria

- Occur within 30 days of procedure.

- Involve only the skin or subcutaneous tissue around the incision.

\section{Plus}

At Least one of the following criteria

- Purulent drainage from the incision.

- Organisms isolated from an aseptically obtained culture of fluid or tissue from the incision.
- At least one of the following signs or symptoms of infection-pain or tenderness, localised swelling, redness or heat and the incision is deliberately opened by a surgeon, unless the culture is negative.

- Diagnosis of superficial incisional SSI by a surgeon or attending physician.

\section{The Following are not considered Superficial SSIs}

- Stitch abscesses (Minimal inflammation and discharge confined to the points of suture penetration).

- Infection of an episiotomy or neonatal circumcision site.

- Infected burn wounds.

- Incisional SSIs that extend into the fascial and muscle layers (see deep SSIs).

\section{DEEP INCISIONAL SURGICAL SITE INFECTIONS}

Deep Incisional Surgical Site Infections must meet the following three criteria

- Occur within 30 days of procedure (or one year in the case of implants).

- Are related to the procedure.

- Involve deep soft tissues, such as the fascia and muscles.

Plus

At least one of the following criteria

- Purulent drainage from the incision, but not from the organ/space of the surgical site.

- A deep incision spontaneously dehisces or is deliberately opened by a surgeon when the patient has at least one of the following signs or symptoms - fever $\left(>38^{\circ} \mathrm{C}\right)$, localised pain or tenderness - unless the culture is negative.

- An abscess or other evidence of infection involving the incision is found on direct examination or by histopathologic or radiological examination.

- Diagnosis of a deep incisional SSI by a surgeon or attending physician.

The SSI rate noted in our study was $3.2 \%$, which is acceptable infection rate for clean surgical procedures. $(29,30)$

\section{CONCLUSION}

Surgical site infections are uncommon in clean surgical procedures and strict adherence to principles of asepsis help in maintaining low infection rate. Antibiotics help in reducing the rate of SSI, but do not eliminate them. A single dose of preoperative antibiotic is sufficient to reduce the risk of postoperative SSI to an acceptable level and prolonged usage of peri-operative antibiotic is not required. Also, usage of single dose antibiotic will be more cost effective in a country like India.

However, role of antibiotic in preventing infections can only be an adjunct in appropriate setting. Overuse of antibiotics with compromise in surgical technique and sterile precautions should not be done.

\section{REFERENCES}

1. Lichtenstein IL, Shulman AG, Amid PK, et al. The tensionfree hernioplasty. Am J Surg 1989;157(2):188-93.

2. Rutkow IM. Surgical operations in the United States; then (1983) and now (1994). Arch Surg 1997;132(9):983-90. 
3. Stephenson BM. Complications of open groin hernia repair. Surg Clin North Am 2003;83(5):1255-78.

4. Law DJ, Mishriki SF, Jeffery PJ. The importance of surveillance after discharge from hospital in the diagnosis of postoperative wound infection. Ann R Coll Surg Engl 1990;72(3):207-9.

5. Glassow F. Is postoperative wound infection following simple inguinal herniorrhaphy a predisposing cause for recurrent hernia? Can J Surg 1964;91(16):870-1.

6. Haley RW, Schaberg DR, Crossley KB, et al. Extra charges and prolongation of stay attributable to nosocomial infections: a prospective interhospital comparison. Am J Med 1981;70(1):51-8.

7. Burke JF. The effective period of preventive antibiotic action in experimental incisions and dermal lesions. Surgery 1961;50:161-8.

8. Polk HC, Lopez-Mayor JF. Postoperative wound infection: a prospective study of determinant factors and prevention. Surgery 1969;66(1):97-103.

9. Stone HH, Hooper CA, Kolb LD, et al. Antibiotic prophylaxis in gastric, biliary and colonic surgery. Ann Surg 1976;184(4):443-52.

10. Yerdel MA, Akın EB, Dolalan S, et al. Effect of single-dose prophylactic ampicillin and sulbactam on wound infection after tension-free inguinal hernia repair with polypropylene mesh. Ann Surg 2001;233(1):26-33.

11. Sanchez-Manuel FJ, Seco-Gil JL. Antibiotic prophylaxis for hernia repair. Cochrane Database Syst Rev 2003;2:CD003769.

12. Waddell TK, Rotstein OD. Antimicrobial prophylaxis in surgery. Committee on antimicrobial agents, Canadian infectious disease society. Can Med Assoc J 1994;151(7):925-31.

13. Tzovaras G, Delikoukos S, Christodoulides G, et al. The role of antibiotic prophylaxis in elective tension-free mesh inguinal hernia repair: results of a single-centre prospective randomised trial. International Journal of Clinical Practice 2007;61:236-9.

14. Ríos A, Rodríguez JM, Munitiz V, et al. Antibiotic prophylaxis in incisional hernia repair using a prosthesis. Hernia 2001;5(3):148-52.

15. Emori TG, Gaynes RP. An overview of nosocomial infections, including the role of the microbiology laboratory. Clin Microbiol Rev 1993;6(4):428-42.

16. Hidron AI, Edwards JR, Patel J, et al. NHSN annual update: antimicrobial-resistant pathogens associated with healthcare-associated infections: annual summary of data reported to the national healthcare safety network at the centers for disease control and prevention, 20062007. Infect Control Hosp Epidemiol 2008;29(11):9961011.
17. Hughes JM, Culver DH, White JW, et al. Nosocomial infection surveillance, 1980-1982. MMWR CDC Surveill Summ 1983;32(4):1SS-16SS.

18. Poggio JL. Perioperative strategies to prevent surgicalsite infection. Clinics in Colon and Rectal Surgery 2013;26(3):168-73.

19. Jones DJ, Bunn F, Bell-Syer SV. Prophylactic antibiotics to prevent surgical site infection after breast cancer surgery. Cochrane Database of Systematic Reviews 2014;3:CD005360.

20. Enzler MJ, Berbari E, Osmon DR. Antimicrobial prophylaxis in adults. Mayo Clinic Proceedings 2011;86(7):686-701.

21. Mangram AJ, Horan TC, Pearson ML, et al. Hospital infection control practices advisory committee guidelines for the prevention of surgical site infection, 1999. Infect Control Hosp Epidemiol 1999;20(4):250-78.

22. Kamat US, Fereirra AMA, Kulkarni MS, et al. A prospective study of surgical site infections in a teaching hospital in Goa. Indian J Surg 2008;70(3):120-4.

23. Berard F, Gandon J. Postoperative wound infections: the influence of ultraviolet irradiation of the operating room and of various other factors. Ann Surg 1964;160(Suppl 2):1-192.

24. American Society of Health-System. Pharmacists ASHP therapeutic guidelines on antimicrobial prophylaxis in surgery. Am J Health Syst Pharm 1999;56(18):1839-88.

25. Antimicrobial Prophylaxis for Surgery. Treatment Guidelines from the Medical Letter 2009;7(82):47-52.

26. Bratzler DW, Houck PM. Antimicrobial prophylaxis for surgery: an advisory statement from the national surgical infection prevention project. Clin Infect Dis 2004;38(12):1706-15.

27. Edwards FH, Engelman RM, Houck P, et al. The society of thoracic surgeons practice guideline series: antibiotic prophylaxis in cardiac surgery; Part I: duration. Ann Thorac Surg 2006;81(1):397-404.

28. Horan TC, Gaynes RP, Martone WJ, et al. CDC definitions of nosocomial surgical site infections, 1992: a modification of CDC definitions of surgical wound infections. Infect Control Hosp Epidemiol 1992;13(10): 606-8.

29. Condon RE, Wittmann DH. The use of antibiotics in general surgery. Curr Probl Surg 1991;28(12):803-7.

30. Woods RK, Dellinger EP. Current guidelines for antibiotic prophylaxis of surgical wounds. Am Fam Physician 1998;57(11):2731-40. 